\title{
Interférométrie XUV sans lame séparatrice : principes et applications
}

\author{
D. Joyeux et F. Polack* \\ Laboratoire Charles Fabry, Institut d'Optique, BP. 147, 91403 Orsay cedex, France \\ ${ }^{*}$ LURE, UMR 130, bâtiment 209D, Campus d'Orsay, 91405 Orsay cedex, France
}

\begin{abstract}
Résumé: La faisabilité d'expériences interférométriques au sens de l'optique classique est liée à la très forte augmentation de la luminance des sources XUV dites cohérente: sources synchrotron, mais aussi laser X et source harmonique. Leur réalisation pratique repose sur la prise en compte des spécificités du domaine XUV, (très petite longueur d'onde, très forte absorption par la matière, cohérence limitee selon les sources) pour la conception et la réalisation d'un interféromètre. Ceci nous a conduit à faire le choix d'interféromètres sans lame séparatrice, utilisant des miroirs plans, si possible sous incidence rasante, du type "miroirs de Fresnel". Cette technique est utilisable dans un très large domaine spectral et a déjà permis de réaliser un certain nombre d'expériences nouvelles dans la région XUV, avec toutes les sources X "cohérentes" disponibles.

Nous discuterons les principes de base, les techniques et contraintes de réalisation d'un interféromètre XUV, ainsi que les applications possibles.
\end{abstract}

\section{REALISATION D'UN INTERFEROMETRE XUV}

Jusqu'à une époque récente, les expériences réalisés en routine à l'aide de sources $\mathrm{X}$ relevaient presque exclusivement de mesures énergétiques, dans lesquelles la partie phase de l'interaction matière - onde n'était pas directement accessible. L'apparition de sources synchrotroniques de très haute luminance, puis l'arrivée du laser $\mathrm{X}$ et de la source harmonique, sont en train de changer cet état de choses, en permettant la réalisation d'expériences d'optique cohérente, c'est à dire où la phase électromagnétique joue un rôle primordial (ex: imagerie limitée par la diffraction, diffraction en champ proche), voire est directement accessible à la mesure (interférences, holographie). On notera que nous n'avons pas évoqué la cohérence propre de la source. En effet c'est bien la luminance spectrale (flux émis par unité de surface normale, par unité d'angle solide, par unité de bande spectrale) qui détermine la faisabilité d'une expérience cohérente donnée, en fixant le flux cohérent disponible; la cohérence nécessaire peut toujours être construite par des moyens optiques simples de type filtrage spatial (pour la cohérence spatiale) ou par filtrage spectral (pour la cohérence temporelle). [1]

\subsection{Les principes de base}

La conception et la réalisation d'interféromètres XUV doivent prendre en compte deux éléments liés au domaine spectral: la faible longueur d'onde, qui accrô̂t les besoins en précision (et parfois en stabilité) des optiques et de leurs assemblages, et la très forte absorption par les matériaux, qui limite (voire interdit, aux énergies VUV) l'utilisation de composants optiques en transmission.

Ce deuxième point nous a conduit à un choix essentiel, celui de renoncer à utiliser un dispositif comportant des lames séparatrices, car celles-ci devraient être ultra-minces, ce qui en renđrait la réalisation avec une planéité de qualité interférométrique au mieux très difficile. Ce choix implique de n'utiliser que des interféromètres "à division de front d'onde", dans lesquels les ondes qui interférent résultent de la division géométrique d'un front d'onde en deux parties, par opposition aux interféromètres "à division d'amplitude", qui utilisent des lames séparatrices. L'inconvénient principal de ce type d'interféromètre est que, au contraire du cas "division d'amplitude", les rayons qui interfèrent sont séparés spatialement dans le front d'onde incident. Celui-ci doit donc présenter une cohérence spatiale suffisante pour que les deux 
rayons présentant la séparation maximale interferent efficacement. Ceci est une contrainte tant que la source utilisée n'est que partiellement spatialement cohérente, ce qui est actuellement le cas de la plupart des sources XUV actuelles, peut-être à l'exception de la source harmonique.

En ce qui concerne la cohérence temporelle nécessaire, elle est déterminée par la différence de marche maximale requise par l'expérience. Dans la pratique (et excepté dans le cas de l'application à la spectrométrie par transformation de Fourier), les différences de marche envisageable sont au plus de quelques $100 \lambda$, c'est à dire une dizaine de microns ou moins dans le meilleur des cas. Ceci impose l'utilisation d'un schéma à bras égaux à quelques dizaines de $\lambda$ près, c'est à dire pratiquement équilibré.

Dans ces conditions, l'interféromètre du type "bimiroir de Fresnel" semble le meilleur candidat, et nous l'avons proposé et mis en cuvre en 1990-1992 [2-3]. Des variantes sont bien entendu possibles, pourvu qu'on en respecte l'esprit: système basé sur des réflecteurs plans, "coupant" la surface d'onde incidente en deux ondes distinctes déviées de façon à se recouvrir en aval des miroirs, et réglés de faȩon à travailler autour de la différence de marche nulle. Ces systèmes sont par principe susceptibles de fonctionner dans une très large bande spectrale, en variant seulement le paramètre de séparation angulaire des faisceaux réfléchis et l'incidence moyenne du faisceau incident. La figure 1 illustre les deux réalisations les plus "basiques" de ce principe, et que nous avons effectivement mises en œuvre.

L'ombre portée couvre la moitié du miroiréchantillon: ceciproduit un champ "référence "et champ "échantillon" dans la figure d'interférence

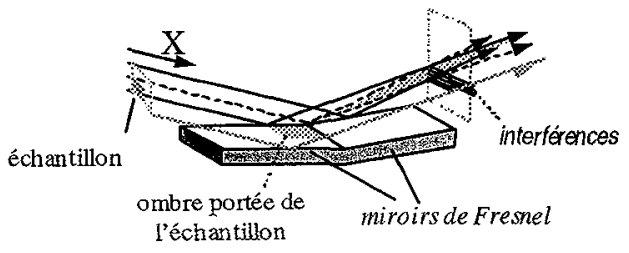

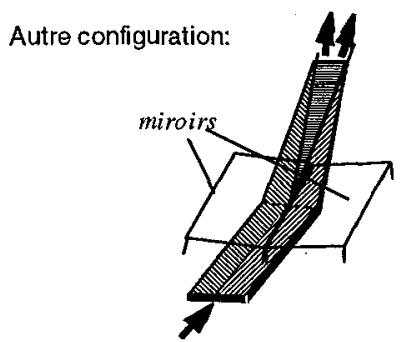

Figure 1: Deux réalisations typiques, que nous avons utilisées dans différentes expériences. Dans les deux cas, les deux demi faisceaux sont déviés de façon à se recouvrir en aval des miroirs. A gauche (miroirs de Fresnel "génériques") la déviation est dans le plan d'incidence, les franges sont perpendiculaire au plan d'incidence. A droite (configuration "livre ouvert"), la déviation est perpendiculaire et les franges parallèles au plan d'incidence. Ces deux configurations, équivalentes au plan des principes, présentent des différences pratiques importantes, tant au point de vue de la formation du champ d'interférence (dépendance angle des faisceaux / angle des miroirs / angle d'incidence) que de la réalisation (surface de miroir active, longueur d'arête active, qualité de forme nécessaire).

\subsection{Contraintes de réalisation}

Les contraintes de réalisation proviennent des techniques de fabrication des éléments optiques (surfaces optiques et assemblages), mais aussi d'éléments relatifs à la mise en œuvre de l'interféromètre, comme la cohérence des faisceaux (spatiale et temporelle), et de la stabilité des montages.

Dans la pratique, les sources XUV et X-mous ne sont que très partiellement cohérentes spatialement (sauf peut-être la source harmonique), et la cohérence nécessaire au niveau de l'interféromètre doit être construite par filtrage spatial. Lorsque c'est nécessaire (cas du synchrotron), la cohérence temporelle est obtenue par filtrage spectral. Ceci ne pouvant être développé davantage dans un court article, le lecteur pourra se reporter pour plus de détails anx manuels, ou à la référence 1 .

Pour ce qui est de l'aspect fabrication, la précision de polissage habitucllement liée à la longueur d'onde (pour la forme et la rugosité) doit être maintenue jusqu'à l'arête commune des deux surfaces optiques. En effet, par le principe même de la division de front d'onde, le dédoublement s'obtient en réglant le faisceau source "à cheval" sur deux miroirs. Du fait de la cohérence spatiale limitée des faisceaux, la zone utile des miroirs est dans la pratique restreinte à une bande dont la largeur va de quelques centaines de microns à quelques millimètres de part et d'autre de l'arête. De plus, pour produire un interfrange de valeur raisonnable, l'angle des faisceaux interférant doit être faible, et il en est de même des basculements relatifs entre miroirs. Par ailleurs, nous avons choisi de "bloquer" le réglage de l'interféromètre à la fabri- 
cation, en collant les surfaces optiques sur une cale de précision (verre poli ou métal rectifié) donnant l'angle des miroirs. Ceci à l'avantage annexe d'éliminer tout problème de vibration ou dilation de l'interféromètre au cours des expériences, mais demande une grande précision dans le positionnement relatif des miroirs, et plus précisément de l'arête commune. On comprend donc que la réalisation de l'interféromètre impose des techniques de fabrication non-standard et de très haute précision [4]

\subsection{Détection}

Alors que le but de l'interférométrie est en général de produire un interférogramme, c'est à dire un système de frange reflétant la répartition ou l'évolution de la phase relative de deux ondes, le mode de détection et d'analyse de l'interférogramme dépend du but recherché. Le plus simple consiste à enregistrer une image du champ de frange (photo, caméra CCD avec ou sans conversion de l'image $X$ en image visible), et à l'analyser ultérieurement. Cette procédure à l'avantage de recueillir un maximum d'informations pour un traitement différé, et l'inconvénient de répartir le flux disponible entre un grand nombre de détecteurs élémentaires (les pixels de l'image). Elle est bien entendu incontournable lorsque l'information est précisément la répartition spatiale d'un paramètre du champ de frange (ex: phase locale des franges dans un contrôle de la surface d'onde produite par un élément optique). A l'autre extrême, il peut être dans certains cas beaucoup plus efficace au point de vue du traitement de l'information de traiter directement l'interférogramme, avant détection photoélectrique, puis de recueillir l'information cherchée sur un nombre restreint de détecteurs. C'est par exemple le cas de la mesure d'indice (cf. \& 2 ci dessous, et ref. 4), ou la mesure consiste à déterminer le déplacement global du champ de franges, et ne nécessite qu'un seul détecteur intégrant tout le flux disponible.

\section{APPLICATIONS}

D'un manière générale, l'interférométrie est une technique qui permet de mesurer directement la phase relative locale de deux ondes électromagnétiques. Or, on sait que la phase électromagnétique est l'un des deux traceurs de l'interaction onde-matière, par l'intermédiaire de la partie réelle de l'indice complexe. Dans le domaine XUV, et à l'exception notable de la diffraction, les physiciens n'ont jusqu'à récemment exploité que l'interaction énergétique (absorption, via la partie imaginaire de l'indice). Bien entendu, ces deux quantités sont liées par la relation intégrale de Kramers-Kronig qui permet de calculer la dispersion spectrale d'indice à partir de celle de l'absorption, mais ce calcul n'est en réalité jamais réalisé dans les conditions idéales, et on sait que le résultat peut même être fortement sujet à caution dans les régions des résonances, les plus intéressantes pour le physicien des matériaux. Il est donc tentant, surtout dans les régions des seuils d'absorption, de réaliser directement des mesures d'indice, ce que permet l'interférométrie. Au minimum, de telles mesures devraient fournir des données indépendantes susceptibles d'être comparées aux sources standards de données. De même, la réflectométrie en énergie est un moyen d'étude couramment employé. Le même argument suggère de mesurer en amplitude et en phase le coefficient de réflexion d'une surface, et non sa seule réflectivité en énergie, ce que permet l'interférométrie, au moins en principe. Par ailleurs, du fait de sa capacité à fournir directement une valeur d'indice à une longueur d'onde donnée, l'interférométrie est très bien adaptée à l'étude de l'influence d'un paramètre extérieur (ex: champ magnétique), à une longueur d'onde donnée.

L'autre grand domaine où l'interférométrie X-UV pourrait s'avérer un outil utile, parfois irremplaçable est celui de "l'optique cohérente XUV" sous toutes ses formes, et en particulier le contrôle des optiques (exemple: objectifs à miroirs pour la microlithographie à $13 \mathrm{~nm}$ ), l'imagerie cohérente XUV (exemple: microscopie interférentielle X), et enfin l'étude des sources XUV non synchrotron par l'intermédiaire de leur cohérence spatiale rayonnée.

Compte-tenu de la place impartie, nous avons choisi de donner un aperçu sommaire de deux expériences typiques des possibilités de la technique interférométrique. La première (mesure d'indice) a été réalisée et est encore en développement, la seconde (spectrographie par transformation de Fourier) est en cours d'étude et fait l'objet d'une thèse.

Nous avons réalisé des déterminations directes d'indice en mesurant le déphasage introduit par un 
échantillon mince traversé par l'un des faisceau de l'interféromètre, en transmission et sous incidence normale. De cette manière, l'influence du terme d'absorption de l'indice complexe est négligeable. Dans le cas de l'interféromètre à bimiroir de Fresnel, la mise en cuvre consiste à introduire dans le faisceau éclairant le bimiroir un échantillon mince limité par un bord droit, de façon qu'il "couvre" l'un des deux miroirs (cf. figure 1) jusqu'à l'arête commune du bimiroir. L'épaisseur de l'échantillon (autosupporté ou sur membrane mince) doit être choisie de façon à produire un déphasage mesurable, et une absorption suffisamment faible pour conserver aux franges un contraste suffisant. Cette expérience a été réalisée pour le carbone autour de $\lambda=4.4 \mathrm{~nm}$ (seuil $\mathrm{K}$ ) et pour l'argent vers $3 \mathrm{~nm}$, avec respectivement $250 \mathrm{~nm}$ et $150 \mathrm{~nm}$ d'épaisseur (donnant des déphasages compris entre 0 et $\lambda / 2$ au maximum). Depuis 1998 , nous utilisons un détecteur spécifique qui fournit directement, avec une sensibilité de l'ordre de 1/300 de frange, le déplacement des franges produit lors de l'introduction de l'échantillon dans l'interféromètre. On obtient un point de la courbe de dispersion d'indice en quelques minutes. La figure 2 illustre les derniers résultats obtenus .
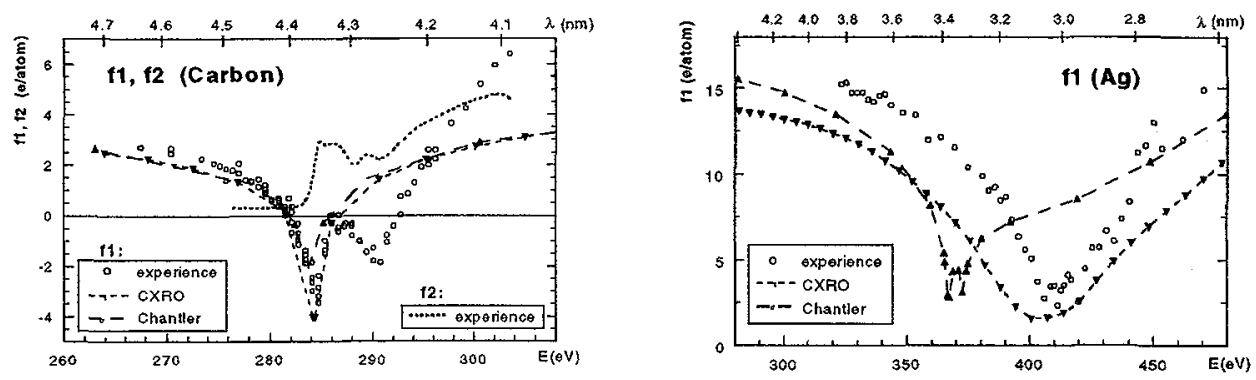

Figure 2: Mesure directe d'épaisseur optique, exprimée en facteur de diffusion atomique (la masse surfacique des échantillons est connue). Voir le texte pour les paramètres des échantillons. Les points marqués CXRO et Chantler sont des données publiées dans la littérature [5,6], obtenues dans le premier cas par le calcul à partir de données d'absorption expérimentales, et dans le deuxième par une modelisation complète du comportement des atomes. Le facteur $\mathrm{f}_{2}$ de l'échantillon de carbone est donné pour information.

La spectromètrie d'absorption par transformation de Fourier est une application très attractive de l'interféromètrie XUV, parce qu'elle permet en principe d'obtenir une grande résolution (plusieurs $10^{5}$ ) et une grande précision dans la détermination des spectres, toutes deux hors de portée des spectromètres à disperseurs dans toute la gamme VUV-X-mous. Bien qu'une réalisation à séparatrice soit possible [7], la technique à division de front d'onde, outre une plus grande simplicité technologique, permet de réaliser des dispositifs utilisables dans un domaine spectral très large sans changement technologique majeur. La réalisation d'un tel interféromètre a été proposée en 1994 [8], et est actuellement étudiée dans notre groupe (collaboration IOTA/LURE, et K. D. Moeller, Fairleigh Dickinson University, USA). L'objectif est d'atteindre une résolution de l'ordre de 2 ou 300000 vers $\lambda=50 \mathrm{~nm}$.

\section{Références}

1. D. Joyeux, P. Jaeglé, A. L'Huillier, in "Current trends in Optics", vol 3, (Academic Press, 1996)

2. F. Polack and D. Joyeux, in X Ray Microscopy III, A.G. Michette, G.R. Morrison and C. J. Buckley, eds., (Springer Verlag, Heidelberg, 1992).

3. J. Svatos, D. Joyeux, F. Polack, D. Phalippou, Optics Letters, 18, 1367 (1993).

4. D. Joyeux, F. Polack, in X-Ray Microscopy and Spectromicroscopy, J. Thieme, G. Schmahl, D. Rudolph, E. Umbach eds., (Springer-Verlag, Heidelberg, 1998).

5. B.L. Henke, E.M. Gullikson and J.C. Davis, Atomic Data and Nuclear Tables 54, 181 (1993)

6. C. T. Chantler, Radiat. Phys. Chem. 41, 759 (1993)

7. M. R. Howells, K. Frank, Z. Hussain, E. J. Moler, T. Reich, D. Möller, D. A. Shirley, Nucl. Instrum. Methods, A347, 182 (1994)

8. F. Polack, D. Joyeux, J. Svatos, D. Phalippou, Rev. Sci. Instrum., 66, 2 (1995) 